\title{
Biodegradable microcontainers - towards real life applications of microfabricated systems for oral drug delivery
}

Abid, Zarmeena; Strindberg, Sophie; Javed, Madeeha M.; Mazzoni, Chiara; Vaut, Lukas; Nielsen, Line Hagner; Gundlach, Carsten; Petersen, Ritika Singh; Müllertz, Anette; Boisen, Anja

Total number of authors:

11

Published in:

Lab on a Chip

Link to article, DOI:

$10.1039 / \mathrm{c} 9 \mathrm{lc00527g}$

Publication date:

2019

Document Version

Peer reviewed version

Link back to DTU Orbit

Citation (APA):

Abid, Z., Strindberg, S., Javed, M. M., Mazzoni, C., Vaut, L., Nielsen, L. H., Gundlach, C., Petersen, R. S., Müllertz, A., Boisen, A., \& Keller, S. S. (2019). Biodegradable microcontainers - towards real life applications of microfabricated systems for oral drug delivery. Lab on a Chip, 19, 2905-2914.

https://doi.org/10.1039/c9lc00527g

\section{General rights}

Copyright and moral rights for the publications made accessible in the public portal are retained by the authors and/or other copyright owners and it is a condition of accessing publications that users recognise and abide by the legal requirements associated with these rights.

- Users may download and print one copy of any publication from the public portal for the purpose of private study or research.

- You may not further distribute the material or use it for any profit-making activity or commercial gain

- You may freely distribute the URL identifying the publication in the public portal 


\title{
${ }_{6}$ Biodegradable microcontainers - towards real life applications of 7 microfabricated systems for oral drug delivery
}

1 Received 00th January 20xx,

2 Accepted 00th January 20xx 3 DOI: 10.1039/x0xx00000x

\begin{abstract}
8 Zarmeena Abid*a,b, Sophie Strindberga,e, Madeeha M. Javed ${ }^{a, b}$, Chiara Mazzoni ${ }^{a, c}$, Lukas Vaut ${ }^{a, c}$, 9 Line Hagner Nielsen ${ }^{a, c}$, Carsten Gundlach ${ }^{d}$, Ritika Singh Petersen ${ }^{a, b}$, Anette Müllertz ${ }^{a, d}$, Anja 10 Boisen $^{a, c}$, Stephan S. Keller ${ }^{a, b}$

11

12 Microfabrication techniques have been applied to develop micron-scale devices for oral drug delivery with a high degree 13 of control over size, shape and material composition. Recently, microcontainers have been introduced as a novel approach 14 to obtain unidirectional release to avoid luminal drug loss, enhance drug permeation, protect drug payload from the harsh 15 environment of the stomach, and explore the ability for targeted drug delivery. However, in order to eventually pave the 16 way for real life applications of these microfabricated drug delivery systems, it is necessary to fabricate them in 17 biodegradable materials approved for similar applications and with strategies that potentially allow for large scale 18 production. In this study, we for the first time evaluate biodegradable microcontainers for oral drug delivery. Asymmetric 19 poly- $\varepsilon$-caprolactone (PCL) microcontainers with a diameter of $300 \mu \mathrm{m}$ and a volume of $2.7 \mathrm{~nL}$ are fabricated with a nove 20 single-step fabrication process. The microcontainers are loaded with the model drug paracetamol and coated with an 21 enteric $\mathrm{pH}$-sensitive Eudragit ${ }^{\circ}$ S100 coating to protect the drug until it reaches the desired location in the small intestine. 22 In vitro dissolution studies are performed to assess the drug load and release profile of the PCL microcontainers. Finally, in 23 vivo studies in rats showed a higher bioavailability compared to conventional dosage forms and confirm the potential of 24 biodegradable microcontainers for oral drug delivery.
\end{abstract}

\section{Introduction}

Among the various conventional ways of drug administration, oral delivery of pharmaceuticals is the preferred route as it offers several advantages. It is non-invasive, provides high patient

30 compliance and is associated with low manufacturing costs. However, the majority of new drug entities entering the market are poorly water soluble which results in a low bioavailability. ${ }^{[1,2]}$ Also, for peptide and protein drugs, oral delivery is a challenge as they can potentially degrade, before reaching their target location in the

35 intestine e.g. due to the low $\mathrm{pH}$ in the stomach or the presence of bacteria or enzymes. ${ }^{[3,4]}$

In the past decades, microfabricated drug delivery systems have been proposed to overcome some of these major challenges in oral drug delivery. ${ }^{[5-8]}$ For this purpose, well-established 40 microfabrication techniques from the semiconductor industry have been applied to reproducibly manufacture microcontainers with precisely controlled dimensions and shapes. ${ }^{[9-11]}$ These devices could potentially provide unidirectional release, control of drug release kinetics and have the ability for targeted delivery of pharmaceuticals in the gastrointestinal tract (GIT). ${ }^{[12,13]}$ The concept of these microcontainers is illustrated in Figure 1. The geometrical structure is cylindrical (Figure 1A) and provides a large surface area that can attach to the mucus layer of the intestinal wall. Drugs in various forms such as powder, liquid or distributed in a drugpolymer matrix have been loaded into the microcontainers (Figure 1B). ${ }^{[14-18]}$ To control the release kinetics and to increase the oral bioavailability of the drug, functional coatings such as a $\mathrm{pH}$-sensitive polymer lids have been applied (Figure 1C) ${ }^{[19,20]}$. When the microcontainers reach the desired location in the GIT, the coating is 55 dissolved and the drug is released followed by intestinal absorption (Figure 1D-E). Several studies have shown that the microfabricated containers can potentially increase the residence time in the intestine and thereby the oral bioavailability of the loaded drug. $[18,19]$ a. The Danish National Research Foundation and Villum Foundation's Center for Intelligent Drug Delivery and Sensing Using Microcontainers and Nanomechanics (IDUN)

b. National Centre for Nano Fabrication and Characterization, DTU Nanolab, Technical University of Denmark, 2800 Kgs. Lyngby, Denmark

Department of Health Technology, DTU Health Tech, Technical University of Denmark, 2800 Kgs. Lyngby, Denmark

d. Department of Physics, DTU Physics, Technical University of Denmark, 2800 Kgs. Lyngby, Denmark

e. Department of Pharmacy, faculty of Health and Medical Sciences, University of Copenhagen, 2100 Copenhagen, Denmark

Electronic Supplementary Information (ESI) available: S1-S5 [See

DOI: $10.1039 / x 0 x x 00000 x$

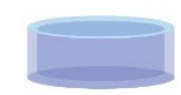

60 A

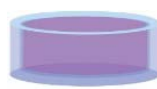

B

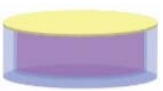

C

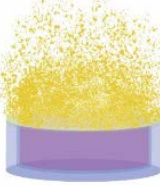

D

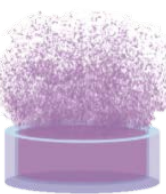

$E$
Figure 1. Schematic representation of the microcontainer concept. A) the microcontainers are fabricated with a bottom and walls, B) a desired drug or formulation is loaded into the microcontainer cavitities, C) a coating is applied in order to protect the drug until

65 release is desired, D) when the microcontainers reach the desired area in the intestine, the lids are dissolved and E) the drug is released 
The first microfabricated drug delivery systems were produced in materials conventionally used in microfabrication such as silicon ${ }^{[22]}{ }_{12}$ 70 poly(methyl methacrylate) (PMMA)[10], and photoresists. ${ }^{[23]}$ However, in the last years there has been a high impetus to fabricate microdevices in biocompatible and biodegradable polymers. $^{[18,19,24,25]}$ DeSimone et al. have introduced the PRINT technique (Particle Replication in Non-Wetting Templates) which 25 75 uses molding in a polymer stamp to produce micron-scale and submicron-scale structures. ${ }^{[26,27]}$ Furthermore, Guan et al. have described a process to produce foldable hydrogels for drug delivery applications. ${ }^{[6]}$ In our center, hot punching has been developed as a simple, low cost and scalable process suitable for the fabrication ofi30

80 individual polymeric microparticles with high structural replication fidelity. ${ }^{[25]}$ In conventional hot embossing, a polymer film is deposited on a carrier substrate and patterned using a stamp. The significant difference of hot punching compared to hot embossing is the introduction of an elastic layer below the actual polymer device

85 layer, allowing discrete particles to be punched out due tols penetration of the residual layer. ${ }^{[25]}$ For the fabrication of microcontainers, hot punching addresses many of the fundamental shortcomings of other methods. It is potentially scalable, enabling the fabrication of large numbers of discrete polymer 90 microcontainers with high aspect ratios using biodegradablet 40 materials suitable for oral drug delivery. The hot punched microcontainers are obtained in ordered arrays solely defined by the stamp design and with the open side of the reservoir pointing upwards which facilitates their handling and further processing

95 such as drug loading. As a major limitation, the fabrication of Poly( 1145 lactic acid) (PLLA) microcontainers, demonstrated earlier, requires multiple processing steps such as spin coating of several polymer layers for the hot punching process. ${ }^{[28]}$ However, spin coating is inherently a batch process, requires preparation of polymer 100 solutions with organic solvents such as acetone or dichloromethane (DCM), and further results in considerable material waste. Furthermore, harvesting of the microcontainers from the carrier substrate has either been based on manual removal or required additional bonding steps for removal from the stamp, which is not 105 suitable for large sample volumes.

Due to the small dimension of the microcontainers, one of the major challenges is to load drug into their cavities. The optimal method should allow for parallel loading of a large number of microcontainers with identical amounts of drug while providing

110 minimal drug waste. In the past, various methods for drug loading1 60 into reservoir-based drug delivery systems have been proposed. ${ }^{[7]}$ Ainslie et al. proposed UV crosslinking of hydrogel matrices with drug. However, the amount of drug that is loaded with this approach is very restricted. ${ }^{[11,12]}$ Alternatively, hot punching in a

115 spin-coated drug-polymer film or supercritical $\mathrm{CO}_{2}$ impregnation of microcontainers filled with polymer have been demonstrated. ${ }^{[20,29,30]}$ In all these methods, solubility of the drug in the polymer matrix or in the supercritical $\mathrm{CO}_{2}$ is required.
Furthermore, the polymer matrix itself will occupy a considerable part of the microcontainer volume, thereby reducing the amount of drug that can be loaded. Typically, drugs are available in powder form acquired from commercial suppliers or prepared as microparticles e.g. using spray drying. Therefore, recently powder embossing has been introduced for reproducible loading of arrays of microcontainers with drug powder. ${ }^{[31]}$ This method provides an excellent loading efficiency, homogeneity, and reproducibility. However, it involves the manual alignment of a shadow mask to the cavity of the microcontainers. This approach is suitable for small experimental studies but not applicable for fabrication of large amounts of drug delivery devices.

Here, we for the first time evaluate the application of biodegradable microcontainers for oral drug delivery. For this purpose, we have developed a novel approach for fabrication of microcontainers in the biocompatible and biodegradable polymer poly- $\varepsilon$-caprolactone $(\mathrm{PCL})$ using a single-step of hot punching. Spin coating of polymer layers on solid carrier substrates was replaced by simple assembly of compression molded polymer films prior to a single step of simultaneous thermal bonding and patterning based on hot punching. This process potentially allows for continuous fabrication of the polymeric devices without the need of organic solvents or expensive batch processing steps. Furthermore, a fast and precise loading of the microcontainer cavities with the model drug paracetamol is demonstrated. Paracetamol was chosen as model drug for this study as it is absorbed in the intestine after oral administration with an oral bioavailability between $70-90 \%$ and there is no absorption though the stomach ${ }^{[32][33]}$. Also, paracetamol is highly water soluble and it is therefore easily released from the microcontainers in aqueous media ${ }^{[34][35]}$. For the purpose of loading the model drug into microcontainers, a modified powder embossing method is implemented where the already existing residual polymer film between the microcontainers replaces the need for the alignment of a shadow mask. The drug-loaded $\mathrm{PCL}$ microcontainers are covered by a $\mathrm{pH}$-sensitive coating of Eudragit ${ }^{\circ}$ S100 applied by spray coating. The microcontainer harvesting step 155 is facilitated by a water soluble substrate of poly(vinylalcohol) (PVA) which is simply dissolved in aqueous medium after microcontainer preparation. The microfabricated drug delivery system is evaluated both in vitro and in vivo to demonstrate the potential for efficient oral drug delivery.

\section{Results and discussion}

The novel fabrication process for biodegradable microcontainers is illustrated in Figure 2A. For the proof of concept, PCL was selected as device material and cylindrical microcontainers with a nominal inner diameter of $230 \mu \mathrm{m}$, a height of $90 \mu \mathrm{m}$ and a reservoir depth 5 of $65 \mu \mathrm{m}$ were designed and fabricated. These dimensions were selected because similar microdevices earlier provided promising drug release kinetics in vitro and improvement of oral bioavailability in vivo. ${ }^{[19-21]}$ 

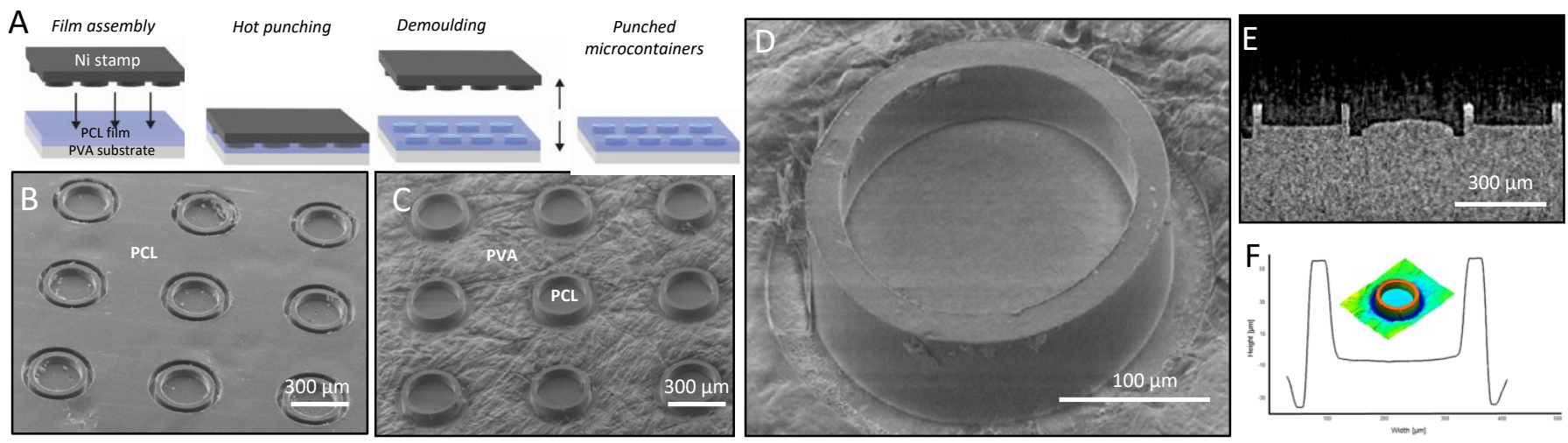

Figure 2. A) Schematic overview of fabrication process: A compression molded PCL film and a PVA substrate are assembled. Hot punching is performed by applying pressure and heat with a Ni stamp. Lastly, a demolding step of the Ni stamp is completed, leading to separation of PCL microcontainers from the surrounding film, B) PCL microcontainer array and surrounding PCL film, C) PCL microcontainer array after removal of the surrounding film, D) Close-up of a single PCL microcontainer on the PVA substrate, E) Cross-sections acquired by $\mathrm{X} \mu \mathrm{CT}$ through the empty microcontainers, F) Optical profile curve and 3D rendering of one single unit for the PCL microcontainer

In this work, compression molding was used to prepare thin PCL 170 device films and PVA substrates. In this process two hot plates function as the molds compressing preheated PCL or PVA pellets into thin films (Supplementary information, S1). This method was introduced to achieve thin and uniform polymer films without the 210 use of solvents, with no waste of material, and to demonstrate the 175 possibility to move from a batch process, such as spin coating, to continuous film preparation using e.g. film extrusion.

The process started with the assembly of a PCL device film and 215 PVA substrate. The device film was then molded by a robust $\mathrm{Ni}$ stamp and finally punched due to the backpressure exerted by the

180 PVA substrate. After the hot punching process was completed, the microcontainers were physically separated from the surrounding PCL film but remained as separate units attached to the underlying2 PVA substrate.

A series of optimization steps were carried out to achieve the 185 desired thickness of the PCL film using compression molding. By varying the processing parameters such as the initial amount o£2 polymer $(0.5-3 \mathrm{~g})$, temperature $\left(60-80{ }^{\circ} \mathrm{C}\right)$ and the hydraulic pressure (20-50 bars), it was possible to adjust the film thickness in the range of 82-103 $\mu \mathrm{m}$. The maximum depth of the structures on

190 the $\mathrm{Ni}$ stamp used in this study was approximately $90 \mu \mathrm{m}$ (Supplementary information, S2). Therefore, a slightly lower23 thickness of the PCL film ensured that the Ni stamp was able to fully penetrate it and reach the PVA substrate during the hot punching process. At the same time, it should ensure complete filling of the 195 stamp features with PCL.

PVA was chosen as the substrate due to its high solubility in aqueous medium, high tensile strength and flexibility. ${ }^{[36]}$ The thickness of the PVA substrate had to be sufficiently high to provide mechanical stability during the punching process and, at the same

200 time, as low as possible to allow its dissolution in aqueous medium 240 in the shortest possible time. The latter was required for harvesting of the microcontainers after completed fabrication where a shorter immersion time in the medium reduces potential loss of drug. In order to control the thickness of the PVA layer, the initial amount of 205 polymer $(5-35 \mathrm{~g})$ and the hydraulic pressure (6-60 bars) were adjusted. Thicknesses in the range of 500-1500 $\mu \mathrm{m}$ were achieved and the final optimal thickness was chosen to be $525 \pm 17 \mu \mathrm{m}$ (SD, $\mathrm{n}=3$ ) as thinner substrates showed mechanical instability and started cracking during the demolding step.

The fabrication of microcontainers was carried out by assembling the $\mathrm{Ni}$ stamp, the PCL device layer and the PVA substrate. In a single step, 1,600 units were punched out arranged in four arrays, each consisting of $20 \times 20$ microcontainers. The optimized temperature for the hot punching process was $70^{\circ} \mathrm{C}$. At this temperature, the PVA substrate still presented mechanical stability and elastic-like properties required for punching of the residual layer due to a glass transition temperature $\left(T_{g}\right)$ for PVA of $85^{\circ} \mathrm{C}$. Furthermore, the hot punching temperature was slightly higher than the melting temperature $\left(\mathrm{T}_{\mathrm{m}}\right)$ for $\mathrm{PCL}$ of $60^{\circ} \mathrm{C}$ and therefore the $\mathrm{PCL}$ device film was in a melted state. Initial optimization was performed by varying the holding time (500-1200 s) and the hydraulic pressure (4-20 bars). With the optimized parameters, PCL microcontainers were successfully punched out and separated from the surrounding $P C L$ film as seen in Figure 2B. The $P C L$ microcontainers adhered well to the PVA substrate as visualized after mechanical removal of the surrounding PCL film in Figure 2C-D and as illustrated by the $\mathrm{x}$-ray microtomography (X- $\mu \mathrm{CT})$ image (Figure 2E). On one hand demolding was possible due to the low surface energy of the Nickel stamp coated with a monolayer of perfluorodexyltrichlorosilane (FDTS). On the other hand, the polar $\mathrm{OH}$ groups of PVA provided excellent compatibility with the ester groups of $\mathrm{PCL}$ leading to exceptional adhesion of $\mathrm{PCL}$ microcontainers to PVA. The hot punching with the optimized parameters resulted in replication of all microcontainers in a single 235 fabrication step and a total process time of $14 \mathrm{~min}$ including holding and embossing time. This was a significant improvement compared to preliminary experiments carried out using a PDMS penetration layer (results not shown). The inner and outer height of the $\mathrm{PCL}$ microcontainers were $64.1 \pm 1.0 \mu \mathrm{m}(\mathrm{SD}, \mathrm{n}=3)$ and $92.0 \pm 1.5 \mu \mathrm{m}(\mathrm{SD}$, $\mathrm{n}=3)$, respectively. The inner diameter was $230.5 \pm 2.2 \mu \mathrm{m}(S D, n=3)$

(Figure 2F), which is very close to the nominal diameter. 
A Mold preparation
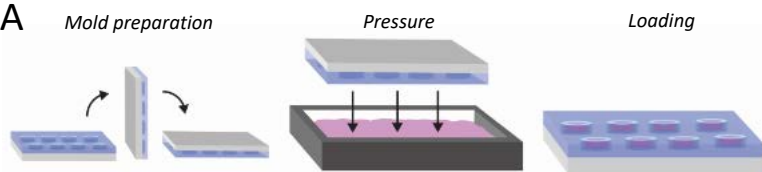

B

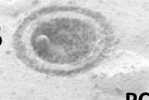

$\mathrm{PCL}$
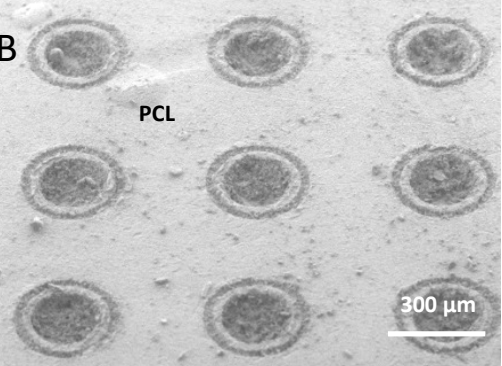
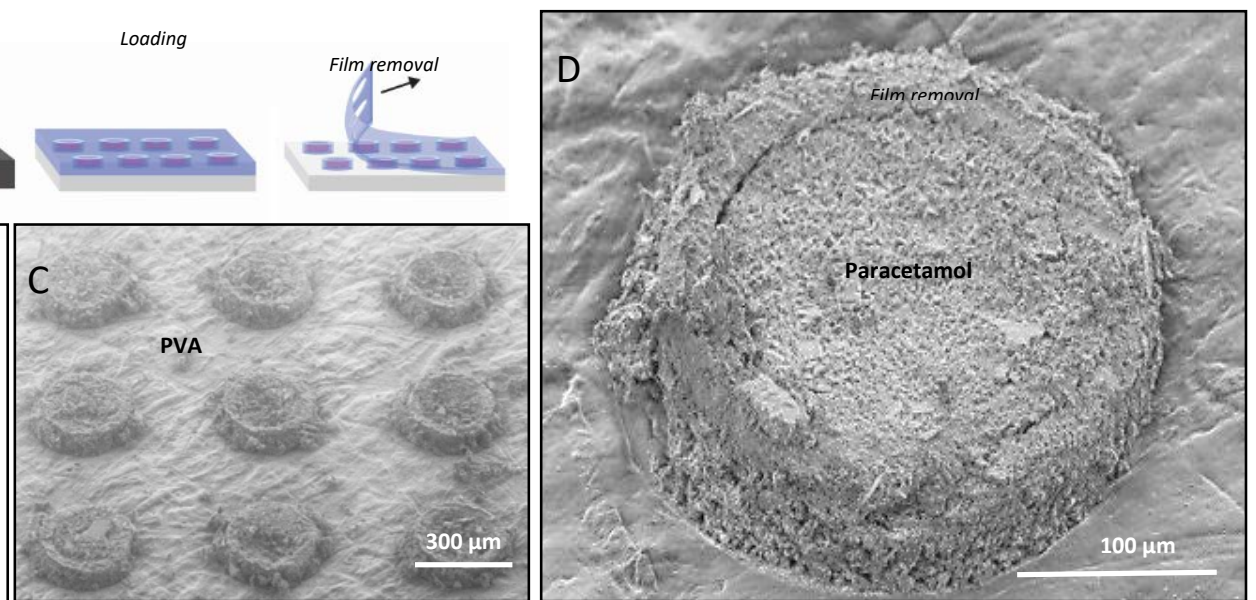

Figure 3. A) Schematic process overview of the drug loading in microcontainers: The PCL microcontainers with the surrounding film are used as mold in the powder embossing process. Paracetamol powder is placed in a holder and pressure is applied. After the pressure is released, the microcontainers are loaded with paracetamol and shadow mask is removed, B) Microcontainer array after the loading process before removal of the surrounding film, C) Microcontainers after the loading process and after removal of the surrounding PCL film, D) Close-up of a single loaded PCL microcontainer

For fabrication of microcontainers, the hot punching process 245 presents several benefits, such as the penetration of the residual layer during a single thermal processing step, no formation of85 residues and no requirement for additional equipment compared to similar attempts using reactive ion etching or laser machining techniques. ${ }^{[33,34]}$ Furthermore, the resulting microcontainers are 250 placed in ordered arrays solely defined by the stamp design and with the cavity of the reservoir pointing upwards which facilitates their handling and further steps such as drug loading.

\subsection{Loading of drug into the PCL microcontainers}

The microcontainer arrays with empty cavities were loaded with 255 paracetamol drug powder. In similar studies, this step required the manual alignment of a shadow mask. ${ }^{[31]}$ Here, the surrounding PC[295 film remaining after the punching process (Figure $\mathbf{2 B}$ ) was used as a stencil to avoid drug deposition in between the microcontainers. Figure 3A shows the overall concept of the drug loading method. 260 The PVA substrate containing the PCL microcontainers and the surrounding $\mathrm{PCL}$ film was used as a stamp for embossing into drug ${ }^{300}$ powder (Figure 3B). After the embossing process, the surrounding $\mathrm{PCL}$ film was removed, limiting drug loading to the container reservoirs. Up to four arrays of 20x20 devices were simultaneously

265 embossed into paracetamol powder both in as purchased and grinded forms. Due to the coarse structure of the powder, grinding05 allowed a more uniform, complete and denser loading (see Supplementary information S3 for SEM images of un-grinded paracetamol loading) of all the microcontainers in the array. As

270 seen in Figure $\mathbf{3 C}$ and $\mathbf{D}$, drug residues between the microcontainers were minimal after removal of the surrounding $\mathrm{PC}^{2} 310$ film.

A successful loading was characterized by drug inside all container cavities. The powder filling required only a few seconds to be 275 performed and resulted in all 1,600 units loaded in one single step. The drug loading was evaluated using microdissolution with array315 of 20x20 devices (Supplementary information S4). Based on the measured dimensions, each PCL microcontainer has a cavity of 2.7 $\mathrm{nL}$ and could therefore theoretically contain up to $3.4 \mu \mathrm{m}$ paracetamol. The measured amount of paracetamol loaded in a single microcontainer was $2.4 \pm 0.1 \mu \mathrm{g}(\mathrm{SD}, \mathrm{n}=5)$, corresponding to a loading efficiency of $71 \%$. The lower actual amount of drug loaded in the microcontainers demonstrates that despite the powder embossing there is still a considerable amount of free volume in each cavity.

\subsection{Enteric coating and harvesting of the drug-loaded PCL 90 microcontainers}

In order to apply the microcontainers for drug delivery in the small intestine, it is desirable that the drug is protected during passage through the stomach. Therefore, the integration of a $\mathrm{pH}$-sensitive coating that only dissolves when the microcontainers reach the small intestine (approximately $\mathrm{pH}$ 7) is required. Enteric polymers have previously been widely investigated for drug delivery systems to overcome the acidic barriers in the stomach. In particular, Eudragit ${ }^{\circledR}$ polymers have been successfully employed in many studies for oral dosage forms. ${ }^{[39]}$ Here, we have combined the advantages of microfabricated drug delivery devices, including controllable size and shape and unidirectional release with the $\mathrm{pH}$ dependent enteric coating which provides a unique system in comparison to existing $\mathrm{pH}$ responsive drug delivery system $\mathrm{s}^{[39][40]}$. Recently, spray coating has been introduced for the deposition of Eudragit ${ }^{\circ}$ films on SU-8 microcontainers and biopolymer microwells loaded with drug. ${ }^{[20][22]}$. These polymeric lids are stable in simulated gastric medium ( $\mathrm{pH}$ 1.6) and dissolve upon immersion in simulated intestinal medium ( $\mathrm{pH} 7.5$ ), triggering the release of the drug. The advantage of this method is that spray coating allows applying a 0 uniform coating on large arrays of microcontainers.

In this work, we implemented spray coating of Eudragit ${ }^{\circ}$ S100 on $\mathrm{PCL}$ microcontainers (Figure 4A). The $\mathrm{pH}$-sensitive coating applied on top of the microcontainers had a thickness of $38 \pm 8 \mu \mathrm{m}(S D, n=5)$. $\mathrm{X}-\mu \mathrm{CT}$ (Supplementary information, S5) and SEM images were used to assess the morphology of the coating after its deposition on the cavity of the microcontainers. Figure $4 \mathrm{~B}$ shows that the coating was uniform and smooth and that the drug-loaded microcontainers were completely covered. For the harvesting of the $\mathrm{PCL}$ microcontainers, the PVA substrate was dissolved. The dissolution 


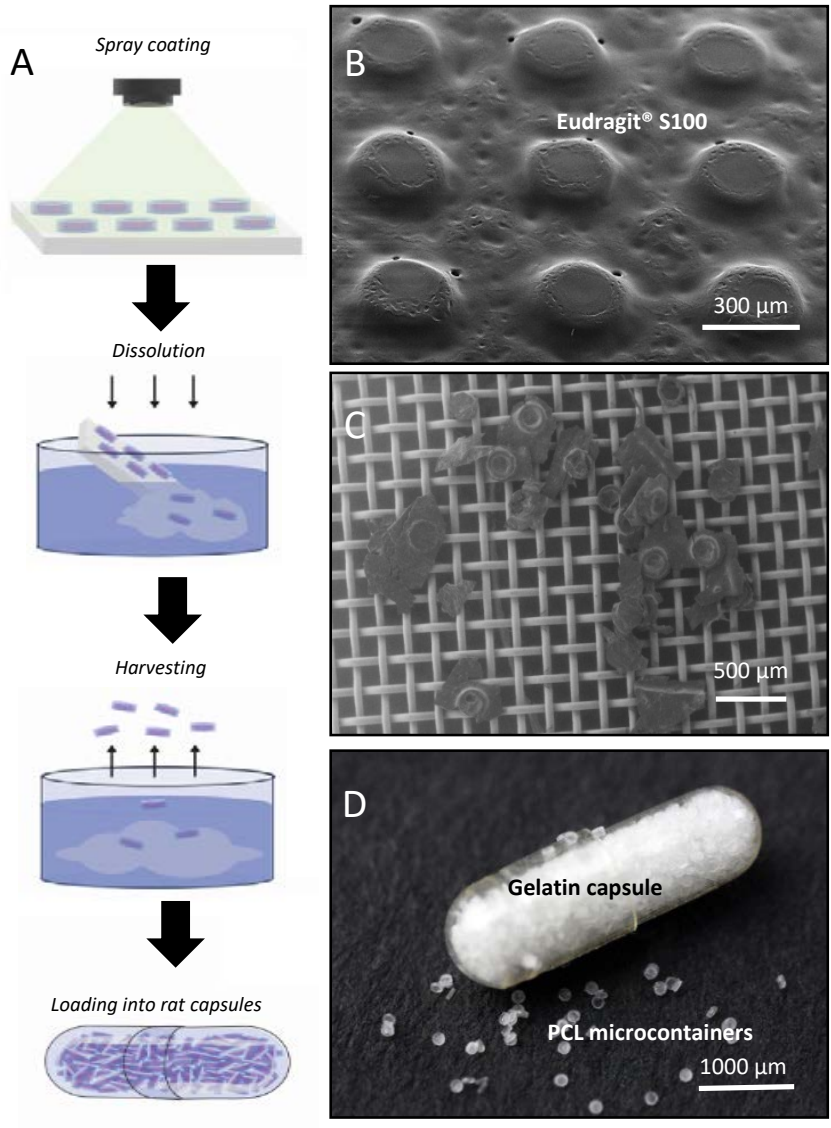

Figure 4. A) Schematic process overview for coating and harvesting microcontainers: $\mathrm{PCL}$ microcontainers on PVA substrate are coated with the $\mathrm{pH}$-sensitive polymer of Eudragit ${ }^{\circ}$ S100. For harvesting microcontainers and dissolving the PVA substrate, the substrate is poured into an aqueous solution. The microcontainers are harvested using a grid and then loaded into gelatin rat capsules B) Microcontainer arrays with an Eudragit S100 coating, C) Harvested microcontainers on a stainless steel grid, D) Rat gelatin capsule loaded with empty microcontainers

320

of the sacrificial PVA substrate was achieved by immersion in acidic aqueous medium ( $\mathrm{pH} 3$ ) for $40 \mathrm{~min}$. Subsequently, the free-floating microcontainers were harvested using a stainless steel filter mesh (Figure 4C) and loaded in rat capsules (Figure 4D).

\subsection{In vitro release of paracetamol from coated PCL} microcontainers

In vitro release studies in a microdissolution profiler were used as a preparative step before the final in vivo studies in order to i) experimentally quantify the amount of drug in each rat capsule and

330 ii) evaluate the performance of the enteric $\mathrm{pH}$-sensitive coating applied on the cavity of the drug-loaded microcontainers.

Rat capsules were prepared for the in vitro studies and each of them were filled with approximately 800 microcontainers. To evaluate the drug release from microcontainers in gastric and 335 intestinal conditions, the in vitro dissolution was completed in two simulated media with a volume of $10 \mathrm{~mL}$. The release of paracetamol was first measured for $30 \mathrm{~min}$ in intestinal medium with $\mathrm{pH}$ 1.6. In this period, $0.25 \pm 0.60 \mathrm{mg}$ paracetamol were 355 released from the microcontainers. This was followed by an 340 investigation of drug release from the gastric medium for $150 \mathrm{~min}$ simulating the transit time in the small intestine. Figure 5 shows that a burst-like release with a significant immediate release of paracetamol was measured for the coated microcontainers. A fast release in the first hour was observed, after which the release curve 345 started saturating. The final amount of drug released after $180 \mathrm{~min}$ showed that each capsule was loaded with $0.72 \pm 0.04 \mathrm{mg}$ paracetamol, which is sufficient drug for in vivo studies. At the same time, this value was lower than expected considering the initial amount of drug loaded in the microcontainers. It is assumed 350 that drug loss mainly occurred during the spray coating of the lids and eventually also during the harvesting of the devices.

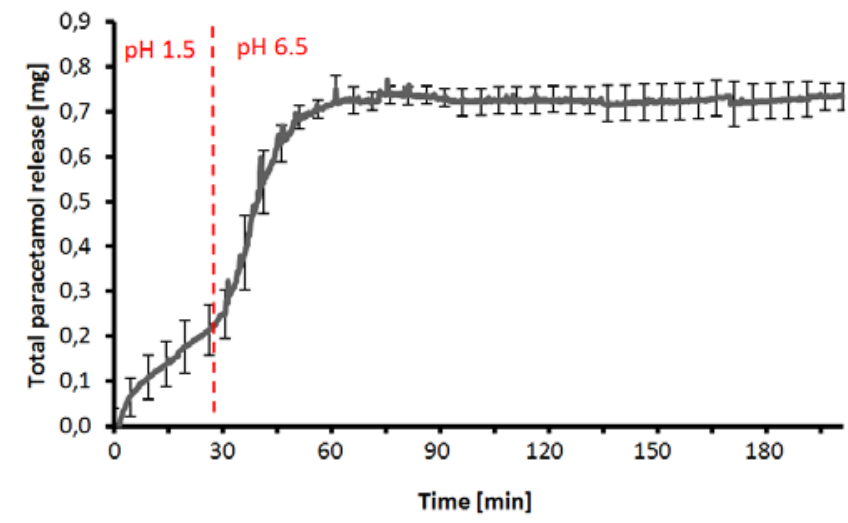

Figure 5. Drug release profiles over time obtained from one capsule of microcontainers filled with paracetamol and coated with Eudragit ${ }^{\circ} \mathrm{S} 100$ in gastric medium at $\mathrm{pH} 1.6$ (from 0 to 30 $\mathrm{min}$ ) and intestinal medium pH 7.5 (30-180 min). Data are presented as mean \pm SD $(n=5)$.
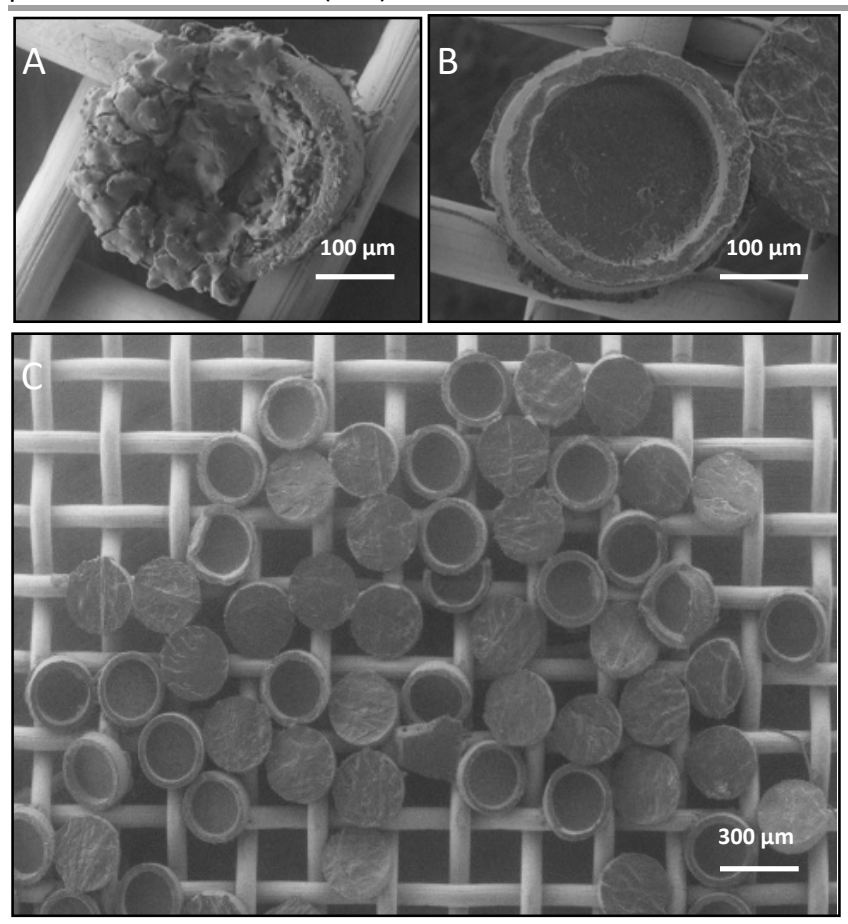

Figure 6. A) Microcontainer after immersion in the gastric medium for $30 \mathrm{~min}, \mathrm{~B})$ close-up of a single microcontainer harvested after $150 \mathrm{~min}$ in intestinal media, C) Microcontainers harvested after $150 \mathrm{~min}$ in intestinal medium 
To verify that the entire drug content was released from the capsules after the dissolution study, the microcontainers wereto investigated in SEM. After immersion in gastric medium for $30 \mathrm{~min}$ (Figure 6A) the enteric coating was still intact and thus the drug was 360 expected to remain inside the microcontainers. For intestinal medium, Figure $\mathbf{6 B}$ and $\mathbf{C}$ confirm that the microcontainers were completely empty after $180 \mathrm{~min}$.

\subsection{Oral pharmacokinetic (PK) study in rats}

In order to study the pharmacokinetic (PK) profile of paracetamol 365 when using PCL microcontainers as vehicle for oral drug delivery, an in vivo study in rats was performed. Rat capsules filled with $\mathrm{PCl}_{4}$ microcontainers, loaded with paracetamol and coated with enteric coating of Eudragit ${ }^{\circ}$ S100, were administered to rats using oral gavage. As a control, rat capsules filled with paracetamol powder 370 and coated with Eudragit ${ }^{\circ}$ S100 were administered. Figure 7 displays the plasma concentration of paracetamol over time after oral dosing of the drug-loaded PCL microcontainers and the control formulation.

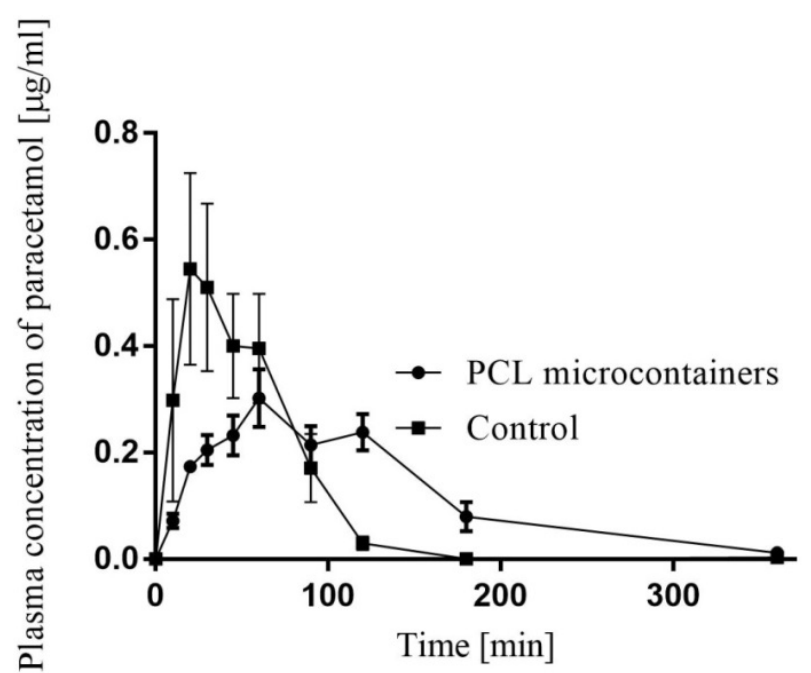

375 Figure 7. Plasma concentrations of paracetamol after oral dosing to rats of either PCL microcontainers filled with paracetamol, coated with Eudragit ${ }^{\circ}$ S100 and filled into gelatine capsules or control capsules filled with paracetamol and coated with Eudragit ${ }^{\circ}$ S100425 The data is presented as mean \pm SEM with $n=7$ for $\mathrm{PCL}$ 380 microcontainers and $n=8$ for control capsules.

Table 1 presents the non-compartmental analysis of the $\mathrm{PK}_{430}$ parameters. The plasma concentration versus time curve shows a 385 fast absorption of paracetamol over the first 90 min for the control formulation. For the PCL microcontainers, it was noted that the profile never reached the same maximum plasma concentrations as the control formulation. This is reflected in the maximum plasma concentration $\left(C_{\max }\right)$, which for PCL microcontainers was found to 390 be $0.3 \pm 0.1 \mu \mathrm{g} / \mathrm{mL}$, whereas it for the control was significantly ${ }^{435}$ higher being $0.9 \pm 0.5 \mu \mathrm{g} / \mathrm{mL}$ (p-value 0.007 ). The time until $C_{\max }$ $\left(T_{\max }\right)$ for PCL microcontainers and the control was $59 \pm 31 \mathrm{~min}$ and $38 \pm 26 \mathrm{~min}$, respectively, and was not significantly different ( $p$ value 0.2371 ). Nevertheless, it still showed a tendency for delayed 395 and sustained absorption of paracetamol released from the $\mathrm{PCL}^{440}$ microcontainers. The sustained absorption might be due to a mucus adhesive effect leading to a prolonged retention of the $\mathrm{PCL}$ microcontainers in the absorptive area of the small intestine. The
PCL microcontainer formulation kept the plasma concentration of paracetamol at a constant level for a longer time period compared to the control. This resulted in an Area Under the Curve $\left(A \cup C_{0-360}\right.$ $\mathrm{min}$ ) for paracetamol of $60 \pm 33 \mu \mathrm{g} \cdot \mathrm{min} / \mathrm{ml}$ for the $\mathrm{PCL}$ microcontainers, and of $39 \pm 18 \mu \mathrm{g} \cdot \mathrm{min} / \mathrm{ml}$ for the control. No significant difference was observed ( $p$-value 0.5973 ), but the results 405 indicate a trend towards a higher $\mathrm{AUC}_{0-360 \mathrm{~min}}$ for the $\mathrm{PCL}$ microcontainers (Table 1).

Table 1. Non-compartmental pharmacokinetic parameters of paracetamol after oral dosing to rats of either PCL microcontainers 0 or the control formulation. Data is presented as mean \pm SEM.

\begin{tabular}{lll}
\hline & $\begin{array}{l}P C L \text { microcontainers } \\
(n=7)\end{array}$ & $\begin{array}{l}\text { Control capsules } \\
(n=8)\end{array}$ \\
\hline $\begin{array}{l}T_{\max } \\
{[\mathrm{min}]}\end{array}$ & $58.6 \pm 31.0$ & $38.1 \pm 26.2$ \\
$\mathrm{C}_{\max }$ & \\
{$[\mu \mathrm{g} / \mathrm{mL}]$} & $0.3 \pm 0.1^{*}$ & $0.9 \pm 0.5^{*}$ \\
$\begin{array}{l}\mathrm{AUG} \text { total } \\
{[\mu \mathrm{g}-\mathrm{min} / \mathrm{mL}]}\end{array}$ & $60.6 \pm 33.5$ & $38.8 \pm 17.8$ \\
\hline
\end{tabular}

*Significant difference after unpaired t-test variance tested with $\mathrm{F}$ test showing significant difference- $p<0.05$

The relative bioavailability was calculated for the paracetamol in 415 the biodegradable PCL microcontainer formulation in relation to the control formulation, and it was found to be $166 \pm 116 \%$. When examining the small intestine and caecum of three rats euthanized $6 \mathrm{~h}$ after dosing by stereomicroscopy (Supplementary information, S6), no PCL microcontainers could be localized. The absence of PCL 420 microcontainers implies that they passed the small intestine at this time.

The in vivo study demonstrates that the biodegradable microcontainers display the same promising features for oral drug delivery as the ones earlier discussed for non-biodegradable microcontainers. ${ }^{[13,20,35]}$ Firstly, the delayed and sustained absorption observed for the PCL microcontainers is similar to results obtained in in vivo rat studies with SU-8 microcontainers filled with amorphous furosemide salt. ${ }^{[21]}$ Secondly, in vivo studies with drug loaded SU-8 or poly(methyl methacrylate) microdevices olso resulted in a higher AUC and relative bioavailability compared to similar controls. ${ }^{[15,20,21]}$

\section{Experimental section}

3.1 Fabrication poly- $\varepsilon$-caprolactone microcontainers: PVA (Mowiflex C17, Kuraray) substrates and PCL $\left(M_{n}=80,000 \mathrm{~g} \mathrm{~mol}^{-1}\right.$, Sigma Aldrich) device films were prepared by compression molding by a hot embosser (Collin ${ }^{\circledR}$ Press, $300 \mathrm{SV}$ ) using the parameters presented in Table 2.

Table 2. Parameters for fabrication of compression molded polyvinyl alcohol substrates and poly- $\varepsilon$-caprolactone device layer

$\begin{array}{llllll}\text { Material } & \begin{array}{l}\text { Amount } \\ {[\mathrm{g}]}\end{array} & \begin{array}{l}\text { Compression } \\ \text { time }[\mathrm{min}]\end{array} & \begin{array}{l}\text { Temperature } \\ {\left[{ }^{\circ} \mathrm{C}\right]}\end{array} & \begin{array}{l}\text { Cooling } \\ \text { ramp } \\ {[\mathrm{K} / \mathrm{min}]}\end{array} & \begin{array}{l}\text { Platen } \\ \text { pressure } \\ {[\mathrm{bar}]}\end{array} \\ & & & & \\ \end{array}$




\begin{tabular}{lllllll}
\hline PVA & 5 & 17 & 150 & 20 & 60 & 500 \\
PCL & 0.5 & 25 & 70 & 20 & 19
\end{tabular}

The compression molded PVA substrates were laser cut (Epilog laser mini, $30 \mathrm{~W}$ ) in squares of $28 \times 28 \mathrm{~mm}$ to fit $4 \times 400_{5}$ microcontainers. The PCL sheets were cut in $28 \times 28 \mathrm{~mm}$ by a scalpel.

445 For the hot punching process, the PCL device film was assembled on top of the PVA substrate. The device film was molded and punched by a robust Nickel stamp $\left(70^{\circ} \mathrm{C}, 500 \mathrm{sec}\right.$., platen pressure at 4 bars). Nickel stamps were fabricated using dry etching and electroplating in a similar manner as described by Petersen et al.[42] After the

450 punching process was finished, the temperature was decreased with a cooling ramp of $20^{\circ} \mathrm{C} \mathrm{min}^{-1}$ to room temperature. Then the stamp was demolded from the polymers. The microdevices were visualized using a Scanning Electron Microscope (SEM). All SEM micrographs were taken by a TM3030Plus Tabletop Microscope 455 (Hitachi, Germany) with a voltage of $15 \mathrm{keV}$ using the SE detector. Optical profiler measurements were performed at DTU Nanolab with PLu Neox 3D optical profilometer (Sensofar, Spain). 20X VSI measurements were conducted on the sample. Stylus profiler (Dektak XTA) measurements were performed to ensure correct

460 height determination by VSI. The data was analyzed using the free SPM data analysis software Gwyddion and the data was levelled with respect to the indentation. Heights were determined based on profiles extracted across the center of the microcontainers. VSt scans were performed near the center and in each of the four 465 corners of the chip. More detailed scans were also made for use in $3 \mathrm{D}$ rendering.

3.2 Loading of drug into the microcontainers: For the loading of microcontainers after container fabrication, the surrounding PCL

470 film was used as a stencil. An electrical compressive press (MTI Corporation, YLI-1-4TA) was used for the drug loading of the microcontainers. For uniform transfer of the powder to the microcontainers, the paracetamol powder was placed in a micro ${ }_{53}$ milled recess with sidelength of $15 \mathrm{~mm}$ and a depth of $1 \mathrm{~mm}$. A

475 constant pressure of $8 \times 10^{-1} \mathrm{~T}$ was applied for $30 \mathrm{sec}$. The powder was embossed inside the container cavities after which pressure and surrounding film were gently removed. The remaining powder was reused for loading of following samples.

480 3.3 Enteric coating deposition: The microcontainers were spraycoated with the $\mathrm{pH}$-sensitive polymer Eudragit ${ }^{\circ} \mathrm{S} 100$ (Evonik, Germany). The solution was prepared mixing $1 \% \mathrm{w} / \mathrm{v}$ Eudragit ${ }^{\circ}$ S100 in isopropanol (Sigma Aldrich, USA). $5 \% \mathrm{w} / \mathrm{w}$ of dibuty 5 sebacate (Sigma Aldrich, USA) in relation to the polymer was added 485 as plastizier. $2 \% \mathrm{v} / \mathrm{v}$ of MilliQ deionized water was added to the final volume. The solution was sprayed over a chip consisting of 400 drug-loaded microcontainers using an ultrasonic spray coater equipped with an accumist nozzle operating at $120 \mathrm{kHz}$ (Sono-Tek USA). During the procedure, the flow rate was kept at $0.1 \mathrm{~mL} / \mathrm{min}$,

490 together with a $1.3 \mathrm{~W}$ generator power. The shaping air was set to 0.02 bars, and the speed of the nozzle was maintained at $10 \mathrm{~mm}$ $\mathrm{sec}^{-1}$, keeping a distance between the tip and the sample of $5 \mathrm{~cm}$. Two alternating spray paths were employed having an offset of $1_{55}$ $\mathrm{mm}$, resulting in a total of 60 passages. The temperature during the 495 spray coating process was kept at $40^{\circ} \mathrm{C}$.

For thickness measurements, clean silicon wafer chips were spray coated with the same Eudragit ${ }^{\circ}$ solution and the same settings. The thickness measurements were performed with a KLA-Tencor Alpha ${ }_{5}$
Step IQ stylus profilometer (Milpitas, USA) with a scan speed of 50 $500 \mu \mathrm{m} \mathrm{s}^{-1}$ and force of $15.3 \mathrm{mg}$. Each chip was measured in three different areas (middle and each side).

3.4 Morphology characterization: The samples as described in Section 4.1 were investigated using a commercial X-ray microtomography system (Zeiss Xradia 410 versa, Germany). The system has an X-ray source operated in reflection geometry, a working high voltage between $40 \mathrm{kV}$ and $150 \mathrm{kV}$ and with a power up to $10 \mathrm{~W}$. Samples were mounted on a flat seam in order to enable alignment to ensure that they were within the field of view 0 in the horizontal plane of the detector. A source voltage of $60 \mathrm{kV}$ and power of $10 \mathrm{~W}$ were used for all measurements in combination with different resolutions settings. Each sample was imaged first with a low resolution using the Large Field of View objective (from $14.5 \mu \mathrm{m}$ to $19.5 \mu \mathrm{m}$ pixel size for different samples and a collection time of $1.5 \mathrm{~h}$, using 1601 projection Images to cover $360^{\circ}$ rotation) in order to observe most of the sample. Then, an area of interest was selected for further investigation with a higher resolution (4.03 $\mu \mathrm{m}$ pixel size and a collection time of $3.5 \mathrm{~h}$ with 1601 projection images covering $360^{\circ}$ rotation) to properly investigate the loading of 25 microcontainers using the ' $4 x^{\prime}$ ' objective. Tomographic data were reconstructed using the commercial software available for the system. The reconstruction software is based on the FDK method which is a filtered back projection algorithm.[43]

3.5: Preparation of rat capsules: Four chips $(4 \times 400)$ of microcontainers were loaded simultaneously and each chip was then coated individually as described above. The solubilization of the sacrificial PVA layer was obtained by soaking the four chips at a time into $600 \mathrm{~mL}$ acidic media $(\mathrm{pH} \mathrm{3})$. After $40 \mathrm{~min}$, the microcontainers were filtered with a stainless steel filter with a mesh opening of $213 \mu \mathrm{m}$ and thickness of $178 \mu \mathrm{m}$ (Spectra/Mesh ${ }^{\circ}$ Woven Filters, Fisher Scientific, Denmark). The microcontainers were dried at $37^{\circ} \mathrm{C}$ for $1 \mathrm{~h}$. Gelatin capsules (Torpac ${ }^{\circ}$, size 9, USA) were filled with individualized microcontainers and weighted before and after filling. The amount of drug inside each rat capsule was estimated based on the microdissolution data. The capsules were weighted before and after loading with microcontainers, and drug loading was normalized to the mean of the total release from the dissolution curve. As controls, capsules were loaded with $0.75 \pm$ $5400.006 \mathrm{mg}$ of grinded paracetamol powder with an amount corresponding to the estimated amount inside the container-loaded capsules. Subsequently, the capsules were coated with a solution of $5 \% \mathrm{w} / \mathrm{v}$ Eudragit ${ }^{\circ} \mathrm{S} 100$ in isopropanol and $5 \% \mathrm{w} / \mathrm{w}$ dibutyl sebacate in relation to the polymer. The capsules were coated by dipping half of it into the coating solution and dried for $15 \mathrm{~min}$ before coating the other half.

3.6: In vitro study: The in vitro release of paracetamol from the microcontainers was tested using a $\mu$-Diss profiler (pION INC, USA) in a similar setup as described elsewhere. ${ }^{[22]}$ Experiments were carried out at $37^{\circ} \mathrm{C}$ employing a stirring rate of $100 \mathrm{rpm}$. The path length of the in situ UV probes was $1 \mathrm{~mm}$, and each channel of the profiler was calibrated with its own standard curve prior to the experiments. The loaded capsules containing either microcontainers or the control powder formulation were poured in $10 \mathrm{~mL}$ Fasted State Simulated Gastric Fluid (FaSSGF pH 1.6, Biorelevant ${ }^{\circ}$, UK) solution after starting the experiment. The experiment was carried out for $30 \mathrm{~min}$ and then the microcontainers were filtered out using a stainless steel grid. Then 60 the medium was changed to $10 \mathrm{~mL}$ Fasted State Simulated 
Intestinal Fluid (FaSSIF pH 7.5, Biorelevant ${ }^{\circ}$, UK) solution in each vial and the experiment was carried out for $150 \mathrm{~min}$.

3.7: Oral PK study in rats: All animal experiments in current study 565 were performed at the Department of Pharmacy, University of Copenhagen, under the license number 2016-15-0201-00892 in agreement with Danish laws regulating experiments on animals and62 EU Directive 2010/63/EU. Sprague Dawley (SD) male rats of an age of 7 weeks (Janvier, France) were acclimatized for 7 days with ad 570 libitum access to water and standard feed. They had a switched light/dark cycle of $12 / 12 \mathrm{~h}$, in a relative humidity of $55 \pm 10 \%$ and a temperature of $22 \pm 1{ }^{\circ} \mathrm{C}$. The rats were fasted for $12 \mathrm{~h}$ prior to dosing with ad libitum access to water and on the day of the study, the weight of the rats was $310 \pm 8 \mathrm{~g}$. The rats were randomly dosed

575 either with one capsule of loaded PCL microcontainers or one capsule containing the control. Each capsule was filled with approximately 800 paracetamol-filled, Eudragit ${ }^{\circ}$ S100 coated PCL microcontainers, corresponding to a dose of $2.8 \pm 0.4 \mathrm{mg} \mathrm{kg}^{-1}$ paracetamol. For the control, capsules coated with Eudragit ${ }^{\circ} \mathrm{S} 100$

580 filled with $2.4 \pm 0.0 \mathrm{mg} \mathrm{kg}^{-1}$ crystalline paracetamol. Each formulation was dosed to 8 rats, and the capsules were dosed by oral gavage with a polyurethrane feeding tube (Instech laboratories Inc., Plymouth Meeting, U.S.). Blood samples ( $200 \mu \mathrm{L}$ per sampling) were retrieved through the tail vein at $0,10,20,30,45,60,90,120$

585180 and $360 \mathrm{~min}$ and collected in ethylenediaminetetraacetic acid (EDTA) tripotassium salt dehydrate coated Microvette -tubes (Sarstedt, Sweden) and centrifuged at $4^{\circ} \mathrm{C}$ at $10,000 \mathrm{rpm}$ for $10 \mathrm{~min}$ to obtain the plasma. The plasma was collected and immediately stored at $-18^{\circ} \mathrm{C}$ until high-performance liquid chromatography

590 (HPLC) analysis. The rats were euthanized and the stomach and intestines were retrieved and immediately stored in $-18^{\circ} \mathrm{C}$ for later analysis by microscopy. For microscopy, the intestine was cut open, divided in $6 \mathrm{~cm}$ sections, placed on glass slide and examined with a stereomicroscope (SteReo Discovery V8, Carl Zeiss Microlmaging $595 \mathrm{GmbH}$, Jena, Germany).

For HPLC, the plasma samples were mixed $1: 1 \mathrm{v} / \mathrm{v}$ ratio with $10 \%$ trichloroacetic acid (TCA) and centrifuged at 10,000 RPM for $10 \mathrm{~min}$ and the supernatant was retrieved for HPLC analysis. The HPLC analyses were carried out on a Dionex Ultimate 3000 pump,

600 equipped with a Dionex Ultimate 3000 autosampler and a UV-VIS lamp. The samples were run isocratic at an absorbance wavelength of $243 \mathrm{~nm}$ at ambient temperature with mobile phase A (Milli-Q water with $0.1 \% \mathrm{v} / \mathrm{v}$ trifluoracetic acid) and $\mathrm{B}$ (acetonitrile) at a $\mathrm{A}: \mathrm{B}$ ratio 95:5 v/v with a Kinetex $5.0 \mu \mathrm{m} \mathrm{C18} 100 \AA$, $100 \times 4.6 \mathrm{~mm}$ 605 column (Phenomenex ApS, Denmark). Injection volume was $20 \mu \mathrm{L}$ at a flow rate of $0.8 \mathrm{~mL} / \mathrm{min}$ with a run time of $10 \mathrm{~min}$ per sample. All results were normalized after the individual dose and the individual weight of each rat. Non-compartmental analysis was applied to determine PK parameters of the paracetamol levels in

610 plasma of each rat. AUC of plasma concentration versus time was calculated by the linear log trapezoidal method and confirmed by column statistics in GraphPad Prism version 7.00 (San Diego, CA, USA). $C_{\max }$ and $T_{\max }$ were determined using the PK profiles. Furthermore, relative bioavailability $\left(\mathrm{F}_{\text {Rel }}\right)$ was calculated by the 615 following equation:

$$
F_{\text {Rel }}=100 \times \frac{A U C_{P C L} \times D_{\text {control }}}{A U G_{\text {control }} \times D_{P C L}}
$$

where $A \cup C_{P C L}$ is the $A \cup C_{0-360 \text { min }}$ of each individual rat dosed with the

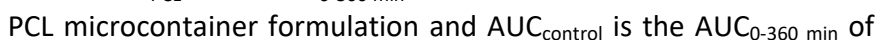

each individual rat dosed with the control formulation, $D_{\mathrm{PCL}}$ is the 620 dose of paracetamol in the PCL microcontainer formulation given to each rat, and $D_{\text {control }}$ is the dose of control formulation for each rat.

The results are shown as mean \pm standard error of the mean (SEM). Statistical significance was tested with an unpaired Student's t-test with Welch's correction if variance was significantly different (tested by F-test) with a p-value $<0.05$ considered as significant.

\section{Conclusions}

The development of oral drug delivery systems for administering drugs in a safe and effective manner in the GIT is of high importance. This study demonstrates the successful fabrication of biodegradable microcontainers using hot punching. The novel process based on assembly of compression molded polymer films avoids the need of solvents or expensive batch processes. In a single processing step, simultaneous patterning of the $\mathrm{PCL}$ and thermal bonding to the underlying PVA substrate is achieved, resulting in replication of large arrays of microcontainers on a water soluble substrate. A modified powder embossing technique has been developed to uniformly load drug into the reservoirs of the microcontainers. This method allows a fast and precise drug loading without the need for alignment of microcontainers to a shadow mask. The fabrication procedure lends itself to continuous manufacturing processes such as in a roll-to-roll (R2R) configuration and is potentially applicable for other polymers and drug formulations. A pH-sensitive lid of Eudragit ${ }^{\circ}$ S100 was applied as a coating on the microcontainers leading to a more targeted system where the drug does not degrade in the harsh gastric conditions before reaching the intestine. In vitro studies confirmed that the drug release is prevented from the acidic environments in the stomach. Finally, this work includes the first in vivo studies of drug absorption from biodegradable microcontainers indicating a higher 50 bioavailability compared to conventional dosage forms, which is an important step towards real life applications of microfabricated drug delivery systems.

\section{Conflicts of interest}

There are no conflicts to declare.

\section{Authors' contribution statement}

Z.A: Conceptualization, Data Curation, Formal analysis, Project administration, Investigation, Methodology, Visualization, Writing original draft, Writing - review and editing. S.A: Data curation, Investigation, Formal analysis, Visualization, Writing - original draft, Writing - review and editing. M.J: Investigation. C.M: Investigation, Formal analysis, Writing - Original draft. L.V: Conceptualization. L.N.: Formal analysis, Supervision, Writing - review and editing. C.G: Data curation, Investigation, Writing - review and editing. R.P: Writing - review and editing, Supervision. A.M: Writing - review 665 and editing, Resources, Supervision. A.B: Funding acquisition, Project administration, Resources, Supervision, Writing - review and editing. S.K: Conceptualization, Supervision, Formal analysis, Funding acquisition, Investigation, Resources, Writing - original draft, Writing - review and editing 


\section{Acknowledgements}

The Danish Research Foundation (project DNRF122) and Villium Fondens Center for Intelligent Drug Delivery (Grant No. 9301) and Sensing Using Microcontainers and Nanomechanics (IDUN) are acknowledged for financial support. Fabrication specialist Lasse 675 Thamdrup is acknowledged for the thorough optical and stylus 30 measurements. Graphical illustrator Ellen Christiansen is acknowledged for the well-illustrated images throughout the paper. The 3D imaging Centre at the Technical University of Denmark is also gratefully acknowledged. Finally, the authors would like to

680 thank Ann-Britt Aspholm-van der Brugge and Kuraray Nordic Ab Oy for straightforward communication, great service and provision of free polymer samples.

\section{Notes and references}

[1] L. S. Taylor and G. G. Z. Zhang, Adv. Drug Deliv. Rev., 2016, 685 101, 122-14

[2] U. Agrawal, R. Sharma, M. Gupta, and S. P. Vyas, Drug Discov. Today, 2014, 19, 1530-1546

[3] J. E. Vela Ramirez, L. A. Sharpe, and N. A. Peppas, Adv. Drug/45 [28] Deliv. Rev., 2017, 114, 116-131

690 [4] C. v H. Laier, B. Gibson, S. Moreno, J. Alberto, T. Rades, S. Hook, L. H. Nielsen, A. Boisen, J. Control. Release, 2019, 294, 91-101

[5] F. J. Martin and C. Grove, Biomed. Microdevices, 2001, $\mathbf{3}_{750}[30]$ 97-107

695 [6] J. Guan, H. He, L. J. Lee, and D. J. Hansford, small, 2007, 3, 412-418

[7] L. H. Nielsen, S. S. Keller, and A. Boisen, Lab Chip, 2018, 18 2348-2358

[8] S. L. Tao and T. A. Desai, Adv. Drug Deliv. Rev., 2003, 55,

700

9] H. D. Chirra and T. A. Desai, Small, 2012, 8, 3839-3846

[10] S. L. Tao, M. W. Lubeley, and T. A. Desai, J. Control. Release, 2003, 88, 215-228

[11] K. M. Ainslie, C. M. Kraning, and T.A. Desai, Lab Chip, 2008 705

[12] K. M. Ainslie, R. D. Lowe, T. T. Beaudette, L. Petty, E. M. Bachelder, and T. A. Desai, Small, 2009, 5, 2857-2863

[13] L. H. Nielsen, S. S. Keller, K. C. Gordon, A. Boisen, T. Rades ${ }_{765}$ and A. Müllertz, Eur. J. Pharm. Biopharm., 2012, 81, 418425

[14] A. Ahmed, C. Bonner, and T. A. Desai, J. Control. release, 2002, 81, 291-306

[15] H. D. Chirra, L. Shao, N. Ciaccio, C. B. Fox, J. M. Wade, $A_{770}$ Ma, and T. A. Desai, Adv. Healthc. Mater., 2014 , 3, 16481654

[16] R. S. Petersen, J. Nagstrup, S. S. Keller, and A. Boisen, Proceedings of the 40th International Conference on Micro and Nano Engineering, 2014

[17] R. Langer and A. Jaklenec, Mater. Sci., 2017, 357, 1138720 1142

[18] S. L. Tao and T. A. Desai, Adv. Mater., 2005, 17, 1625-1630

[19] L. H. Nielsen, J. Nagstrup, S. Gordon, S. S. Keller, J. $\varnothing_{\text {stergaard, T. Rades, A. Müllertz, and A. Boisen, Biomed }} 780$ Microdevices, 2015, 17, 1-7
25 [20] C. Mazzoni, F. Tentor, S. A. Strindberg, L. H. Nielsen, S. S: Keller, T. S. Alstrøm, C. Gundlach, A. Müllertz, P. Marizza, and A. Boisen, J. Control. Release, 2017, 268, 343-351

[21] L. H. Nielsen, A. Melero, S. S. Keller, J. Jacobsen, T. Garrigues, T. Rades, A. Müllertz, and A. Boisen, Int. J. Pharm., 2016, 504, 98-109

[22] A. B. Foraker, R. J. Walczak, M. H. Cohen, T. A. Boiarski, C. F. Grove, and P. W. Swaan, Pharm. Res., 2003, 20, 110-116

[23] S. L. Tao and T. A. Desai, Nano Lett., 2007, 7, 1463-1468

[24] J. Nagstrup, S. Keller, K. Almdal, and A. Boisen, Microelectron. Eng., 2011, 88, 2342-2344

[25] R. S. Petersen, S. S. Keller, and A. Boisen, Lab Chip, 2015, 15, 2576-2579

[26] J. P. Rolland, B. W. Maynor, L. E. Euliss, A. E. Exner, G. M. Denison and J. M. DeSimone, Journal of the American Chemical Society, 2005, 127, 10096-10100

S. E. a Gratton, S. S. Williams, M. E. Napier, P. D. Pohlhaus, Z. Zhou, K. B. Wiles, B. W. Maynor, C. Shen, T. Olafsen, E. T. Samulski, and J. M. DeSimone, Acc. Chem. Res., 2008, 41, 1685-1695

R. S. Petersen, R. Mahshid, N. K. Andersen, S. S. Keller, H. N. Hansen, and A. Boisen, Microelectron. Eng., 2015, 133, 104-109

[29] R. S. Petersen, S. S. Keller, and A. Boisen, Macromol. Mater. Eng., 2017, 302, 1-6

P. Marizza, S. S. Keller, A. Müllertz, and A. Boisen, J. Control. Release, 2014, 173, 1-9

Z. Abid, C. Gundlach, O. Durucan, C. v. H. Laier, L. H. Nielsen, A. Boisen, and S. S. Keller, Microelectron. Eng., 2017, 171, 20-24

L. F. Prescott, Br. J. clin. Pharmac., 1980, 10, 291-298

J. A. H. Forrest, J. A. Clements, and L. F. Prescott, Clinical Pharmacokinetics, 1982, 7, 93-107

[34] L. R. Shaw, W. J. Irwin, T. J. Grattan and B. R. Conway, Drug Development and Industrial Pharmacy, 2008, 31, 515-525

760 [35] E. Szaek, A. Karminska, D. Murawa, K. Potom, B. Urbaniak, M. Sobiech, E. Grzeskowiak, T. Grabowski, A. Wolc, Z. J. Kokot and P. Murawa, Pharmacological Reports, 2011, 63, 1519-1525

[36] T. S. Gaaz, A. B. Sulong, N. A. Majid, A. A. H. Kadhum, A. B. Mohamad, and A. A. Al-amiery, Molecules, 2015, 20. 22833-22847

S. J. Pearton and D. P. Norton, Plasma Process. Polym., 2005, 2, 16-37

[38] J. Y. Shiekh-Ahmad, Machining of Polymer Composites, 2009, Springer

[39] B. Luppi, F. Bigucci, V. Zecchi and T. Cerchiara, Drug Delivery, 2009, 16, 24-29

[40] H. Du, M. Liu, X. Yang, and G. Zhai, 2015, Drug Discov. Today, 20, 1004-1011

25 [41] L. H. Nielsen, T. Rades, B. Boyd, and A. Boisen, Eur. J. Pharm. Biopharm., 2017, 118, 13-20

[42] R. S. Petersen, S. S. Keller, O. Hansen, and A. Boisen, J. Micromechanics Microengineering, 2015, 25

[43] L. A. Feldkamp, Opt. Soc. Am. A, 1984, 1, 612-619 